\title{
Student Reading Strategies of GIS Workflow Diagrams
}

\author{
Zdena Dobesova \\ Department of Geoinformatics, Faculty of Science, Palacký University, Olomouc, Czech Republic \\ zdena.dobesova@upol.cz
}

Keywords: education, eye-tracking, workflow, GIS, visual programming languages, ArcGIS.

Abstract. This paper describes the results of eye-tracking testing of workflow diagrams from ArcGIS ModelBuilder. The respondents were 26 students of study branch Geoinformatics and Geography at Palacky University. The article describes that the reading patterns could be detected as aggregation from scanpath of respondents. The aggregation of trajectories is better that to explore the individual respondent scanpath separately. The combination of both methods helps to discover the complicated behavior of respondents. Punctually exploring of eye tracking outputs brought evidence that the student reading strategies are influenced by the arrangement of workflow diagrams, especially by the orientation. The main orientation of diagram determines the main reading flow. In fact, the respondents do not start reading at the starting graphical elements and also the flow of reading ends earlier than the end of the diagram is. In the case of more branches of the diagram, the top (or left) branch is read more carefully than the second branch of the diagram.

\section{Introduction}

The group of students of bachelor study branch Geoinformatics and Geography attendees at the fourth semester the subject "Programming 2". The first three lessons students have lectures about the creation of workflow diagrams in component ModelBuilder for ArcGIS for Desktop. The creation is easy by "drag and drop" the graphical elements into the workflow diagram. The sequence of processes is designed as a chain of graphical elements in the graphical editor. Yellow rectangles represent an operation; the blue and green ovals represent input and output data to/from operations (Fig. 1).

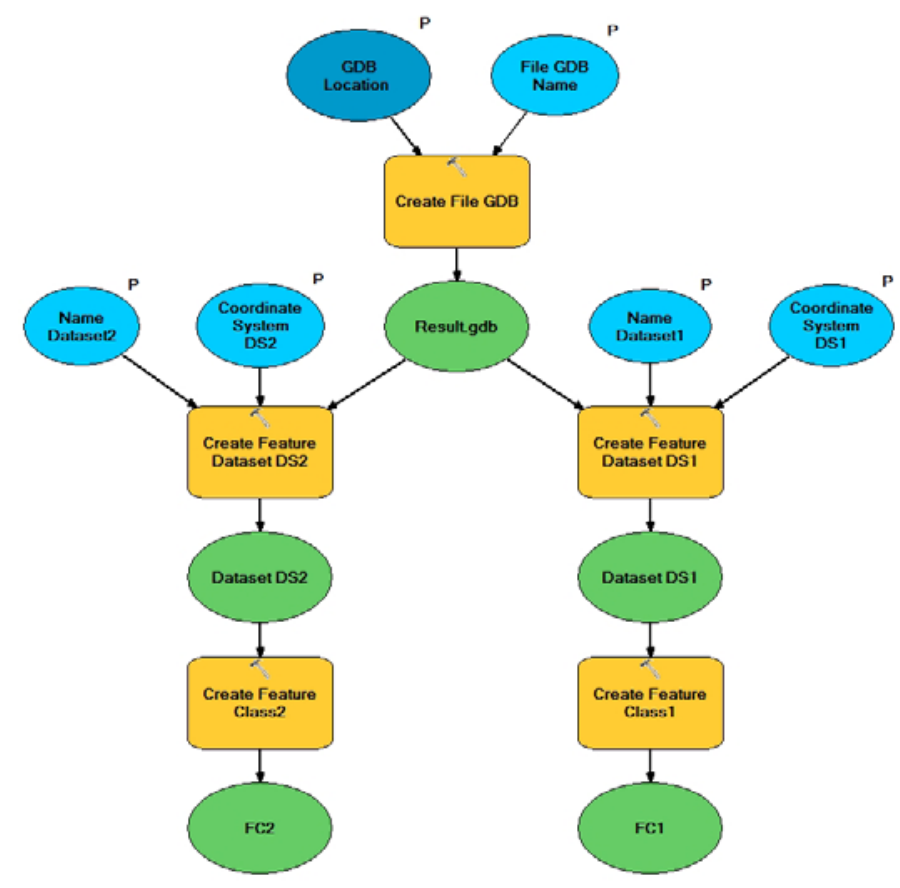

Figure 1. Example model from ModelBuilder with top-down orientation 
Workflow diagrams belong to the sort of visual programming languages [1]. Diagram expresses the steps of spatial processing data in GIS. The workflow diagram is called model in ModelBuilder [2]. The term model will be used for the next part of article instead term workflow diagram.

The students start with the simple models with three to five elements with simple functionality. They are focused on the correct function of the model. The lecturing continues with using extended possibilities as inserting iterators, for repetitive processing data, inserting inner variables, parameters, and nested (sub) models. The students are able to create a huge advanced model e.g. with 25 or more elements.

The subject "Programming 2" is supported by e-learning portal. There are presentations with a description of steps how to create various models. Moreover, there are data for practical examples and assignments [3]. Students submit totally four assignments with various models to e-learning portal.

Besides the functionality of the models, the quality of their layout affected the understanding and comprehension of the model. Students are encouraged to use the Auto Layout button that automatically align the graphical element to the grid, set the same distance between elements and automatically redraw the connector lines. The teacher encourages students to use Auto Layout button from the first model to adopt it as a habit after any remake of the model.

Students are novices in the design of models at the beginning of the semester. Subsequently, after the intensive practising, they are skilled users. The research question was "How students read the models?". And a lot of other questions appeared: "Do students differ in reading models? Has the arranging of models influence on reading strategy? How the reading strategy is influenced by the task that is solved above model?". To answer these questions, we prepared eye tracking test with 41 models as stimuli.

\section{Eye-tracking Experiment}

The Department of Geoinformatics provides the specialised eye-tracking laboratory with eye tracker equipment SMI RED 250. For the design of the experiment, the software SMI Experiment Suite $360^{\circ}$ was used for the experiment. The resolution of the monitor to record eye movement was $1920 \times 1200$ pixels. The sampling frequency was $250 \mathrm{~Hz}$.

The experiment consisted of two parts where the second part follows immediately the first one. The models in the first part were showed without any task or question. This form of eye-tracking testing is called "free-viewing". The student could read models freely. The displaying time was fixed to 7 seconds of each stimulus for each student. The first part contained 19 models. The stimuli were interleaved by fixation stimulus with a small black cross in the centre of the screen. This stimulus assures the same start centre position of student's eyes before next stimulus and the sane for all participants. This is necessary to provide a comparison of the reading strategy between students and aggregate the scan-path.

In the second part of the eye-tracking experiment, the models were accompanied by tasks. One task was above one model. The tasks were showed in the form of sentence on a previous stimulus before displaying model. After task reading, the fixation cross was displayed in the centre of the screen (for the same purpose as in the first part). The respondents fulfil the task by mouse click(s) in the area of the model to any graphical element. The task was e.g. "Click on all output data." In some cases, the correct answer was one click (e.g. one output data). In other cases, the correct answer was two or more mouse clicks (e.g. more output data). The second part of the experiment with models consists of 22 stimuli. The same stimuli were used in the first and in the second part. The using or the same stimulus was intended to compare the reading strategy. Four models were used twice with different tasks in the second part of the experiment.

The number of respondents was 27 ( 5 female and 22 male). The age was from 20 to 25 . One respondent was removed caused by bad calibration and low tracking ratio. All participants were at the same level of knowledge. The testing group was homogenous. The participant variation is influenced only by the personal capabilities of perception and cognition. Participant sample size is sufficient 
according to [4] where 10 participants are the minimum size. More participants and more stimuli prevent from making a mistake and give better statistical power.

\section{Reading Strategies of Models in Free-viewing Test}

The testing of perception, cognition, reading and comprehension of graphic outputs is the aim of many types of research. It could be prepared as a simple questionnaire with questions that could be presented in a print form or on the monitor. The objective measures of results could be both the score of correct and wrong answers both the total time of the task. These numerical results are used in several investigations for cartographic outputs [5], business processing models [6] and UML diagrams [7].

The latest investigations also used the eye-tracking methods for exploring the style of pupil reading the printed text [8], UML diagrams [9] and cartography [10]. Eye-tracking is used as an objective empirical study of student reading behaviour.

We evaluated the reading behaviour of a student on a model from both parts of the test: the first free-viewing and the second task-solution viewing. Our hypothesis was that the reading behaviour would be different for the same models from the first and second parts. Next hypothesis was that the reading patterns are mainly influenced by the main orientation of flow of model. Opposite expectation was that reading has no patterns and looks like "random walk" (from any point to any point). In that case, there is no discernible pattern.

Firstly, the separate scan path of each student was explored. For display of measured data, the open source software OGAMA v. 5 was used [11]. For a demonstration of reading patterns, the model with orientation left to right was chosen. This model has two horizontal branches on the right side. It is interesting to identify the process of reading of these parallel branches. In next pictures are presented both scan path of one reader and the aggregation of saccades for 26 students.

One typical scan path of one student P15 is showed in Fig. 2. This is scan path for 7 seconds in the first free viewing part ( 7 seconds is the same for all). The red numbers in circles show the order of eye fixation. The red connector lines draw the movement of gaze (saccades). Many other students have similar scan path like this. Only some students were "slow readers" and gaze was only recorded on the left part of the model, they do not reach the end of the model. On another hand, some students went twice through the model. After the first scan, they went back from right end to the left start part of the model. We assume that the time 7 second is enough for free-viewing part of the average user.



Figure 2. Typical scanpath of one student in free-viewing test

The typical reading pattern could be described like this: It is started in the top left corner (Fig. 2 fixation $2,3,4,5,6,7)$. The gaze continued from left to the right following the upper branch of the model (fixation 14, 15, 16). Interesting is the gap and the skip to the end of the branch (fixation 17, 18). The students do not read each graphical symbol systematically. The move to the bottom 
horizontal branch is in the middle (fixation 21, 22). The reading of bottom branch is not started at green oval where the branch out is. The number of fixation is lower on the bottom branch than on upper branch.

The description of separate scan path does not bring such clear evidence of reading patterns. To identify reading patterns we used the method Flow map. That method aggregates all scanpath based on automatically generated Thiessen polygons. Aggregations are displayed as a map of arrows where thickness represents the amount of moving from one aggregation center to another aggregation center. Software V-Analytics was used for calculation Flow map [12]. Software V-Analytics is formerly intended for exploring geographic movement data. The authors of this software successfully tested its applicability to eye tracking data and the capability to extract useful knowledge about users [13].

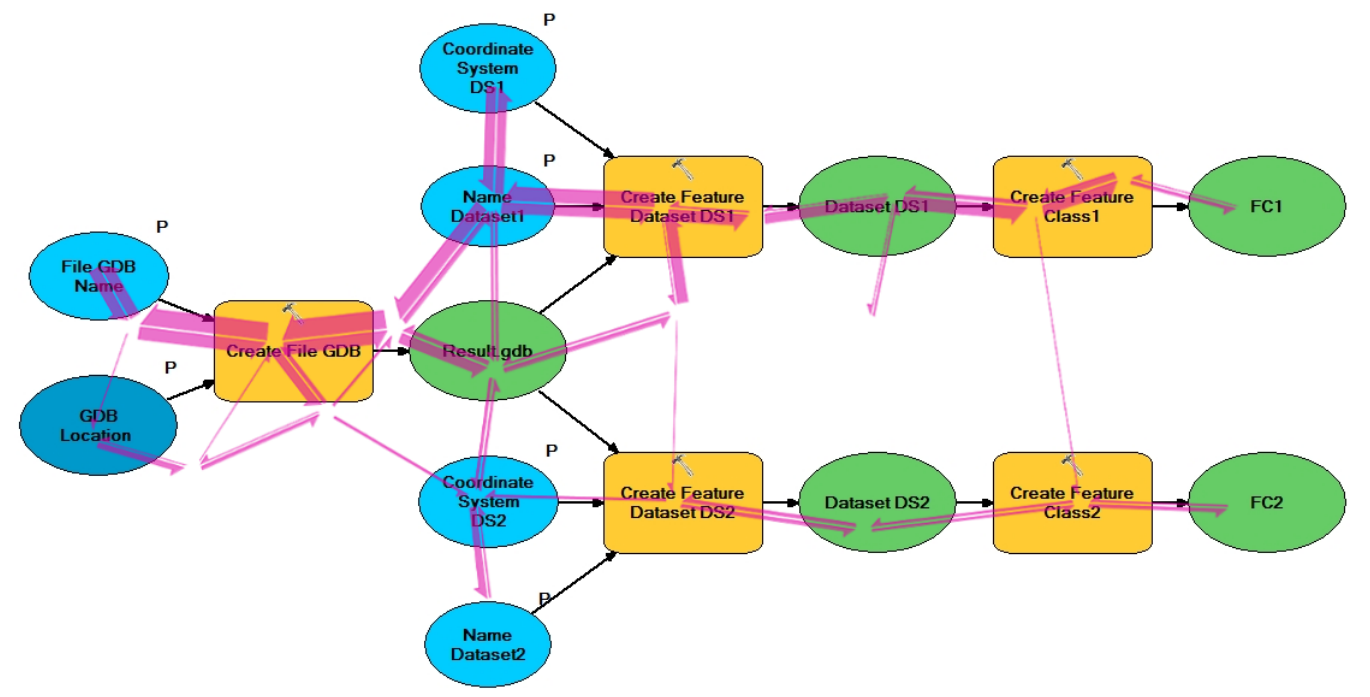

Figure 3. Aggregations of the student scanpaths for model in free-viewing test

Fig. 3 displays the aggregation of all scanpath by violet arrows for the same diagram (as Fig. 2) for 26 students. The aggregated lines that have less than five movements were removed from Flow map to better view the pattern. There is evident that the main flow of reading is at the left part (but not reach both blue input data as strongly as a first yellow box with the operation). More scan paths are on the upper horizontal branch than on the lower horizontal branch. The three thin violet arrows between both branches show the places where gaze cross between branches that are various for users.

\section{Reading Strategies of Models Task-solving Test}

The same model was used in the task-solving eye-tracking test. Task was: "Select all operations where the input data is geodatabase Result.gdb." In that case, the scanpath is totally different. Fig. 4 shows the scan part of the same student P15 (as Fig. 2). More fixations are concentrated near the green oval that represents the geodatabase Result.gdb. Other fixations are on the two yellow operations where places of correct answers are. The task was not so easy because firstly was necessary to locate the geodatabase and subsequently follow the connecting lines to find the correct operations. Student nearly does not read the left and right symbols on the edge. The fixations are concentrated around the correct solutions. 




Figure 4. Typical scanpath of one student in task solving test

Also, Flow map of aggregated scanpath was calculated for evaluation of behavioural patterns in task testing. Fig. 5 shows that students read the left and central part carefully. The most moves are near the correct answer, a lot of moves are between both yellow boxes with correct operation. There is evident that user several times compare the relation between input geodatabase and operations. But the reading is also partly influenced by the orientation of the model. There is evident that reading starts at the left starting part of the model not matter the task (user do not know, where is the correct answer at the beginning of the test). The reading pattern is not so influenced by the flow of model as in the free-viewing test. But some more than five visits are at both horizontal branches.

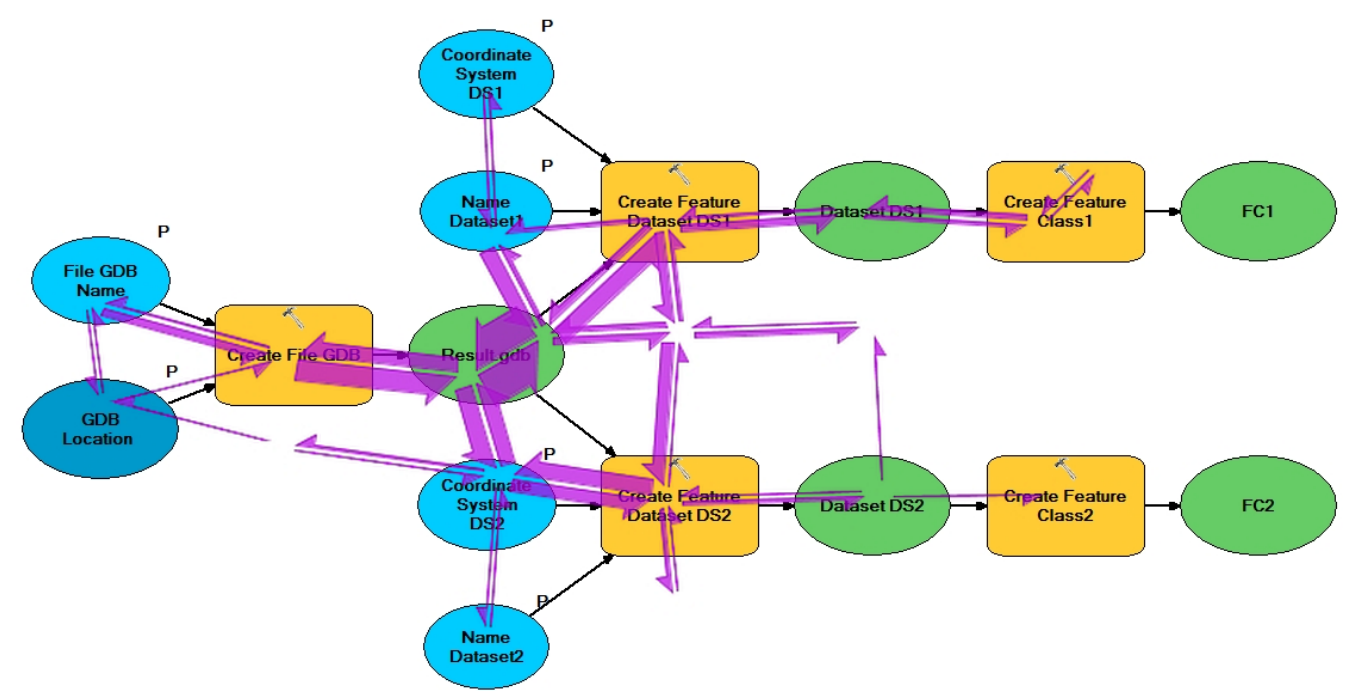

Figure 5. Aggregation of the student scanpaths for model in task-oriented test

The patterns like these presented were discovered also at the other models that were tested. Both separate scan path of users both Flow maps verify the same reading patterns in models. The reading of branches was tested in also a top-down oriented model like Fig. 1 (function are the same as the model in Fig.2). The similar skips between vertical branches were recorded like in the case of horizontal branches.

\section{Summary}

The article shows some patterns in the reading model from ModelBuilder. The reading strategy is presented in the article on one model in two reading parts. The results are the same in testing simple or more complicated models. The students do not have a various reading strategy; the strategy is nearly the same for all. The "random walk" has not been identified. The exploring of separate user 
scanpath and exploring the aggregated scanpath in the form of Flow map together brings visual interpretation how to look like reading behaviour. In both tests (free-viewing and task-solved) the students are influenced by the flow of model. The reading strategy starts at the top - left corner and follows the main flow of diagram to the right end (bottom respectively when top-down orientation is dominant). In free-viewing without any task, the model structure is followed in more systematic way. In the case of more branches the upper branch (vertical orientation of model) has a dominant reading with more fixation than a bottom branch. In case long branches some of the graphical elements are skipped. Readers often skip between long branches before reading them to the end. So the orientation of model is not followed so strictly. In task-oriented tests the reading patterns, that follow the main flow of diagram, is influenced by the location of the correct answer. More transition of gaze is located here. But assure the correct answer the user go through model repetitively, went back to the start and shortly scan all model. The method Flow map from V-Analytics is helpful in visualisation and discovering the reading patterns of the models.

\section{Acknowledgements}

This article has been created with the support of project CZ.1.07/2.3.00/20.0170.

\section{References}

[1] Z. Dobesova and P. Dobes, "Differences in visual programming for GIS," vol. 519-520, ed. Switzerland: Trans Tech Publications, 2014, pp. 353-356.

[2] Esri. (2015). What is ModelBuilder? Available: http://desktop.arcgis.com/en/arcmap/10.3/analyze/modelbuilder/what-is-modelbuilder.htm

[3] Z. Dobesova, "E-learning for visual programming language," IEEE 12th International Conference on Emerging eLearning Technologies and Applications, 2014, pp. 103-108.

[4] K. Holmqvist, M. Nyström, R. Andersson, R. Dewhurst, H. Jarodzka, and J. Van de Weijer, Eye tracking: A comprehensive guide to methods and measures: Oxford University Press, 2011.

[5] A. Pődör and M. Kiszely, "Experimental investigation of visualization methods of earthquake catalogue maps," Geodesy and Cartography, vol. 40, pp. 156-162, 2014/10/02 2014.

[6] K. Figl, J. Mendling, and M. Strembeck, "The influence of notational deficiencies on process model comprehension," Journal of the Association of Information Systems, vol. 14, pp. 312-338, 2013.

[7] H. Störrle, "On the impact of layout quality to understanding UML diagrams: Diagram type and expertise," in Visual Languages and Human-Centric Computing (VL/HCC), 2012 IEEE Symposium on, 2012, pp. 49-56.

[8] M. Malčík, E. Mechlová, Z. Sikorová, and A. Mentel, "Analysis of visual perceptual patterns on screen using eyetracker technology," in Emerging eLearning Technologies and Applications (ICETA), 2014 IEEE 12th International Conference on, 2014, pp. 311-317.

[9] H. Störrle, N. Baltsen, H. Christoffersen, and A. M. Maier, "On the Impact of Diagram Layout: How Are Models Actually Read?," in MODELS, 2014.

[10] S. Popelka, A. Brychtová, and V. Voženílek, "Eye-tracking and its use for assessment of maps," Geografický časopis, vol. 64, pp. 71-87, 2012.

[11]A. Voßkühler, "OGAMA Open Gaze And Mouse Analyzer," http://www.ogama.net/, 2014.

[12]G. Andrienko and N. Andrienko. (2015). V-Analytics (a.k.a. CommonGIS). Available: http://geoanalytics.net/V-Analytics/ 
[13]G. Andrienko, N. Andrienko, M. Burch, and D. Weiskopf, "Visual analytics methodology for eye movement studies," IEEE Transactions on Visualization and Computer Graphics, vol. 18, pp. 2889-2898, 2012. 